\title{
LAS CONDICIONES DE GOBERNANZA PARA LA IMPLEMENTACIÓN DE LAS CONTRIBUCIONES NACIONALMENTE DETERMINADAS: EL CASO DEL PERÚ ${ }^{1}$
}

\author{
Ivan Lanegra \\ Pontificia Universidad Católica del Perú
}

\begin{abstract}
Resumen:
La presente ponencia propone un marco de análisis para la dimensión de la gobernanza en la implementación en el Perú de las Contribuciones Nacionalmente Determinadas derivadas del Acuerdo de París (NDC por sus siglas en inglés). Los países menos desarrollados, como el señalado, agregan a las dificultades tecnológicas y de financiamiento para implementar las NDC, las debilidades institucionales. En dicho contexto, el análisis de las condiciones de gobernanza debe considerar los factores políticos de corto plazo, las constricciones al desarrollo institucional, así como elementos de economía política. Esto nos lleva a considerar tanto la dinámica sectorial y territorial, como los distintos papeles que los actores con poder económico y político juegan alrededor de la política climática.
\end{abstract}

\section{Palabras clave:}

Cambio climático, Acuerdo de París, Contribuciones Nacionalmente Determinadas, gobernanza.

\footnotetext{
1 Ponencia presentada en el Foro Internacional de Ciencias Sociales Cambio Climático. Desastres y Gobernanza realizado en Cusco del 2 al 5 de agosto de 2017.
} 


\title{
The Conditions of Governance for the Implementation of the Nationalally Determined Contributions: The case of Peru
}

\begin{abstract}
:
This paper proposes a framework of analysis for the governance dimension in the implementation in Peru of the Nationally Determined Contributions derived from the Paris Agreement (NDC). The less developed countries, such as the one mentioned, add to the technological and financing difficulties to implement the NDC, the institutional weaknesses. In this context, the analysis of governance conditions should consider short-term political factors, constraints on institutional development, as well as elements of political economy. This leads us to consider both the sectoral and territorial dynamics, as well as the different roles that actors, with economic and political power, play around climate policy.
\end{abstract}

\section{Keywords:}

Climate change, Paris Agreement, Nationally Determined Contributions, governance.

\section{Ivan Lanegra}

Abogado por la Pontificia Universidad Católica del Perú (PUCP) y egresado de la Maestría en Ciencia Política y Gobierno por la misma universidad. Es miembro del Grupo de Estudios Ambiente y Sociedad - GEAS— de la PUCP.

Profesor de Ciencia Política en la PUCP y en la Universidad del Pacífico. Ha sido coordinador de Arreglos Institucionales del Proyecto de Apoyo a la Gestión del Cambio Climático de Libélula Instituto para el Cambio Global. Actualmente es miembro del Consejo Técnico-Consultivo del Servicio Nacional de Certificación Ambiental para las Inversiones Sostenibles (SENACE).

Correo: ilanegra@pucp.edu.pe 


\section{Introducción}

La implementación de las Contribuciones Nacionalmente Determinadas (NDC), en el marco del Acuerdo de París (ONU 2015), plantea un desafío para los países menos desarrollados; a las dificultades tecnológicas y de financiamiento se agregan las debilidades institucionales. Por dicha razón, las estrategias que guíen dicho esfuerzo en el ámbito nacional deben ser analizadas bajo el concepto de gobernanza climática. Siendo el concepto de gobernanza (governance) polisémico (Porras 2016) es necesario empezar con una definición operativa. Kooiman definióla gobernanza como «los arreglos por los cuales actores públicos y privados tratan de resolver problemas sociales o crear oportunidades sociales, y cuidan las instituciones [...] dentro de las cuales se llevan a cabo las actividades del gobierno» (Porras 2016: 62). La gobernanza es —en términos generales - una nueva forma de dar dirección a la acción social o colectiva que consiste en la coordinación intersectorial para el logro de objetivos comunes, cuestión vinculada a su vez con la reforma institucional, con la interacción de las nuevas redes de actores e instituciones, la innovación en las políticas públicas, incluyendo el desarrollo de respuestas nuevas a los viejos problemas de coordinación y rendición de cuentas.

Como ha señalado Jeffrey Sachs, «la buena gobernanza y el imperio de la ley producen un sentimiento de seguridad y bienestar. En cambio, la corrupción, la anarquía, la falta de políticos fiables, la desigualdad en el acceso a los servicios públicos, la discriminación, la información privilegiada en los negocios y demás generan mucha infelicidad» (2015: 31). Para ello, Sachs propone cinco principios de gobernanza. En primer lugar, los actores - sean estatales o privados- deben responder por las consecuencias de sus decisiones o acciones. Para esto, en segundo lugar, es necesario conocer dichas decisiones y acciones y los procesos que llevaron a ellas; es decir, es indispensable la transparencia. En tercer lugar, es necesario que los ciudadanos $-\mathrm{y}$ en general todos los involucrados por las decisiones o accionespuedan participar (o influir) en la toma de decisiones. En cuarto término, los actores deben internalizar el costo o los beneficios de sus decisiones y acciones, partiendo del principio de «no hacer 
daño». Finalmente, debe entenderse que el desarrollo sostenible plantea deberes universales, por lo que ningún gobierno o actor puede estar al margen de dichas responsabilidades (Sachs 2015).

Por otra parte, como describe Porras (2016), son cinco los problemas fundamentales que debe enfrentar un buen modelo de gobernanza. En primer lugar la cooperación entre actores - gubernamentales o no-,locual presupone que cuestiones como el cambio climático no pueden ser resueltas por un solo actor, incluso si hablamos del Estado; entonces, es necesario entender en qué consiste la cooperación entre los distintos actores involucrados, cómo es posible alcanzarla y mantenerla en el tiempo, así como sus implicaciones para cada uno de dichos actores. El segundo problema fundamental es la presencia de la autoorganización como respuesta a la cooperación, poniendo el énfasis en los mercados y en la sociedad civil, con lo cual los ciudadanos, las OSC (Organizaciones de la Sociedad Civil) y algunos otros actores e instituciones comparten el poder que antes parecía más concentrado o consolidado en menos manos. Este poder, sin embargo, no es formal ni duro, sino más difuso y suave (Porras 2016).

El tercer problema es la rendición de cuentas, el cual en un contexto de cooperación alcanza una mucha mayor amplitud; la rendición de cuentas tiene diversas expresiones, incluyendo la político electoral (democrática), la administrativa (burocrática), la personal (ética), la profesional (ética profesional), la de resultado - output- (satisfacción del cliente) y la deliberativa (diálogo público). El cuarto problema es la efectividad de la acción colectiva, en un contexto de cooperación y dispersión del poder. Por último, el quinto problema es la interdisciplinariedad, en tanto la cooperación obliga a acercarse y escuchar los puntos de vista existentes en distintas disciplinas que sirven de base para la toma de decisiones de cada uno de los actores. Es en el marco de estas condiciones más complejas en el cual debe construirse la gobernanza climática.

\section{La gobernanza del cambio climático}

Dentro del marco descrito, la gobernanza climática constituye una parte de la gobernanza en general que tiene por propósito cumplir plenamente los objetivos de la política climática. Su base es la institucionalidad climática. Es decir, todas aquellas políticas públicas, procesos, sistemas y redes de colaboración y coordinación —-formales e informales - que las entidades con responsabilidades ante el cambio climático utilizan para normar, planificar y gestionar eficientemente sus actividades, así como para coordinar con otros actores - estatales o no-. Dicha institucionalidad conforma a su vez la base 
para la gestión de la política climática. Desde luego, tanto la política climática, como la gobernanza climática y la gestión de la política climática están en constante sinergia y tensión con sus pares de las esferas económica, social y ambiental (Lanegra 2017).

¿Cuáles son los principales desafíos que la dimensión de la gobernanza plantea a la implementación de las NDC en el Perú?, ¿qué políticas son necesarias y cómo darles viabilidad? Es un lugar común hablar de la debilidad del Estado y de las instituciones. La pregunta que planteamos es, en cambio, ¿cuáles son las oportunidades existentes para fortalecer la gobernanza climática? Para ello debemos considerar factores políticos de corto plazo, así como las constricciones al desarrollo institucional que han generado el tipo de crecimiento económico que tenemos en el Perú. Esto nos lleva a comprender tanto la dinámica territorial, como los distintos papeles que juegan los actores con poder económico y político. La presente ponencia busca proponer tanto un marco de análisis como una primera aproximación al caso peruano a partir de dicho marco.

La gobernanza climática mantiene una relación de ida y vuelta con respecto a los objetivos de la política climática. La gobernanza forma parte de las condiciones para alcanzar los objetivos; a la vez, los objetivos deben diseñarse considerando las condiciones de gobernanza existentes y los cambios que en dicha esfera pueden ocurrir en el corto plazo. Pero, como recuerda Mirta Geary (2016), no todos entienden la política y la gobernanza del mismo modo (incluyendo su variante climática). Algunos defienden el enfoque de la modernización ecológica, la cual sostiene que los problemas climáticos pueden resolverse mediante la tecnología, poniendo el acento en la innovación tecnológica y el uso de las mejores tecnologías - menos contaminantes-, haciendo uso del análisis costo-beneficio al momento de establecer las prioridades de la política climática. (Geary 2016).

Un segundo enfoque, el de la ecología política, es crítico con la visión de la modernización ecológica arriba descrita, señalando sus límites. La ecología política pone el acento en el carácter conflictivo de la cuestión climática, aspecto que queda oculto cuando los actores traducen cada problema a términos monetarios o cuando los actores tienen una confianza ciega en la tecnología como solución a los problemas climáticos. Desde esta mirada, los conceptos no son inocentes y pueden traer consigo sesgos en medio de su aparente neutralidad. Ante ello plantean mostrar los límites de la racionalidad económica mediante los principios de la sustentabilidad ecológica - que enfatiza los límites físicos del planeta- y la equidad social -que presta especial atención a los problemas de justicia y distribución de las cargas climáticas-. En resumen, la política y gobernanza climáticas deben, desde luego, tomar en cuenta tanto la ciencia —en particular la ecología-, 
la tecnología y la economía; pero también deben poner sobre la mesa los problemas de equidad y justicia distributiva (Geary 2016). El enfoque elegido — sea más técnico, más político o algún tipo de combinación de ellos-influirá sustancialmente en el modelo de gobernanza a utilizar. Desde nuestro punto de vista, la ecología política constituye una mirada que responde mejor a los problemas que plantea la gobernanza, lo cual no implica dejar de lado la relevancia de la tecnología y de la economía, en tanto también determinan oportunidades y límites para aquella. Ahora toca centrarse en el objetivo de la política climática.

\section{Las contribuciones nacionalmente determinadas}

El Acuerdo de París establece como objetivo no superar el límite de $2^{\circ} \mathrm{C}$ de aumento de la temperatura global por encima del promedio preindustrial, un objetivo laxo en relación con las exigencias de la comunidad científica, la cual apostaba por un objetivo de $1,5^{\circ} \mathrm{C}$. De otro lado, el acuerdo reza que el mismo «se aplicará de modo que refleje la equidad y el principio de las responsabilidades comunes pero diferenciadas y las capacidades respectivas, a la luz de las diferentes circunstancias nacionales» (ONU 2015). En tal sentido, los objetivos del acuerdo, conforme su Artículo 3, deben alcanzarse a través de las Contribuciones Nacionalmente Determinadas (NDC por sus siglas en inglés). Así, «en sus contribuciones determinadas a nivel nacional a la respuesta mundial al cambio climático, todas las Partes [del Acuerdo de París] deberán realizar y comunicar los esfuerzos ambiciosos [...] con miras alcanzar el propósito del presente Acuerdo [...]. Los esfuerzos de todas las Partes representarán una progresión a lo largo del tiempo, teniendo en cuenta la necesidad de apoyar a las Partes que son países en desarrollo para lograr la aplicación efectiva del presente Acuerdo» (ONU 2015). No obstante, el Acuerdo de París deja abierta la puerta a un esfuerzo progresivo con miras a alcanzar esta última meta, incluyendo una actualización quinquenal que ayude a ajustar el nivel de ambición nacional de cada uno de los países. Queda por ver, desde luego, si los esfuerzos por reducir los gases de efecto invernadero en este esquema flexible son lo suficientemente rápidos o si llegan demasiado tarde. Este diseño flexible, en comparación con el diseño del Protocolo de Kioto, es el que hizo viable políticamente el logro del tratado internacional.

Las NDC del Perú en materia de mitigación contemplan una reducción del $30 \%$ con respecto a las emisiones de gases de efecto invernadero proyectadas para el año 2030, como parte de un escenario business as usual (BaU), es decir, en el supuesto de que las tendencias actuales no varíen. El 
Estado peruano estableció que dos terceras partes de dicha reducción serán no condicionadas y una tercera parte sí. La parte no condicionada será implementada a través de inversiones y gastos con recursos internos, públicos y privados. La parte condicionada, en cambio, estará supeditada a la disponibilidad de financiamiento externo internacional y a las condiciones favorables.

El Perú ha establecido su reducción de emisiones partiendo del escenario BaU, construido tomando como base el año 2010, y luego proyecta una trayectoria de emisiones crecientes hasta el año 2030. Las proyecciones totales consideran las emisiones y las remociones del sector uso de suelo, el cambio de uso de suelo y la silvicultura (USCUSS) - donde está el problema de la deforestación-; sin embargo, también comunicó las emisiones, tanto en la base como en la meta, sin considerar las emisiones de dicho sector. Las proyecciones de las emisiones al 2030 aparecen en el siguiente cuadro.

Cuadro 1

\begin{tabular}{|c|c|c|}
\hline & $\begin{array}{c}\text { Emisiones } \text { MtCO }_{2} \text { eq incluyendo } \\
\text { USCUSS }\end{array}$ & $\begin{array}{c}\text { Emisiones } \text { MtCO }_{2} \text { eq sin incluir } \\
\text { USCUSS }\end{array}$ \\
\hline 2010 & 170,6 & 78,0 \\
\hline 2030 & 298,3 & 139,3 \\
\hline
\end{tabular}

Fuente: República del Perú 2015.

En síntesis, el Perú se compromete a reducir en un 30\% (o en un 20\% sin condiciones) las emisiones previstas en el año 2030, es decir, casi 90 (o 60) MtC02eq. Finalmente, el gobierno peruano comunicó la reserva de su derecho de actualizar este escenario conforme disponga de mejor información sobre las emisiones y sus tendencias antes de 2020.

El Perú también ha considerado en sus NDC acciones de adaptación. En el siguiente cuadro se recogen los cinco sectores que han sido priorizados en virtud de sus niveles de vulnerabilidad. 


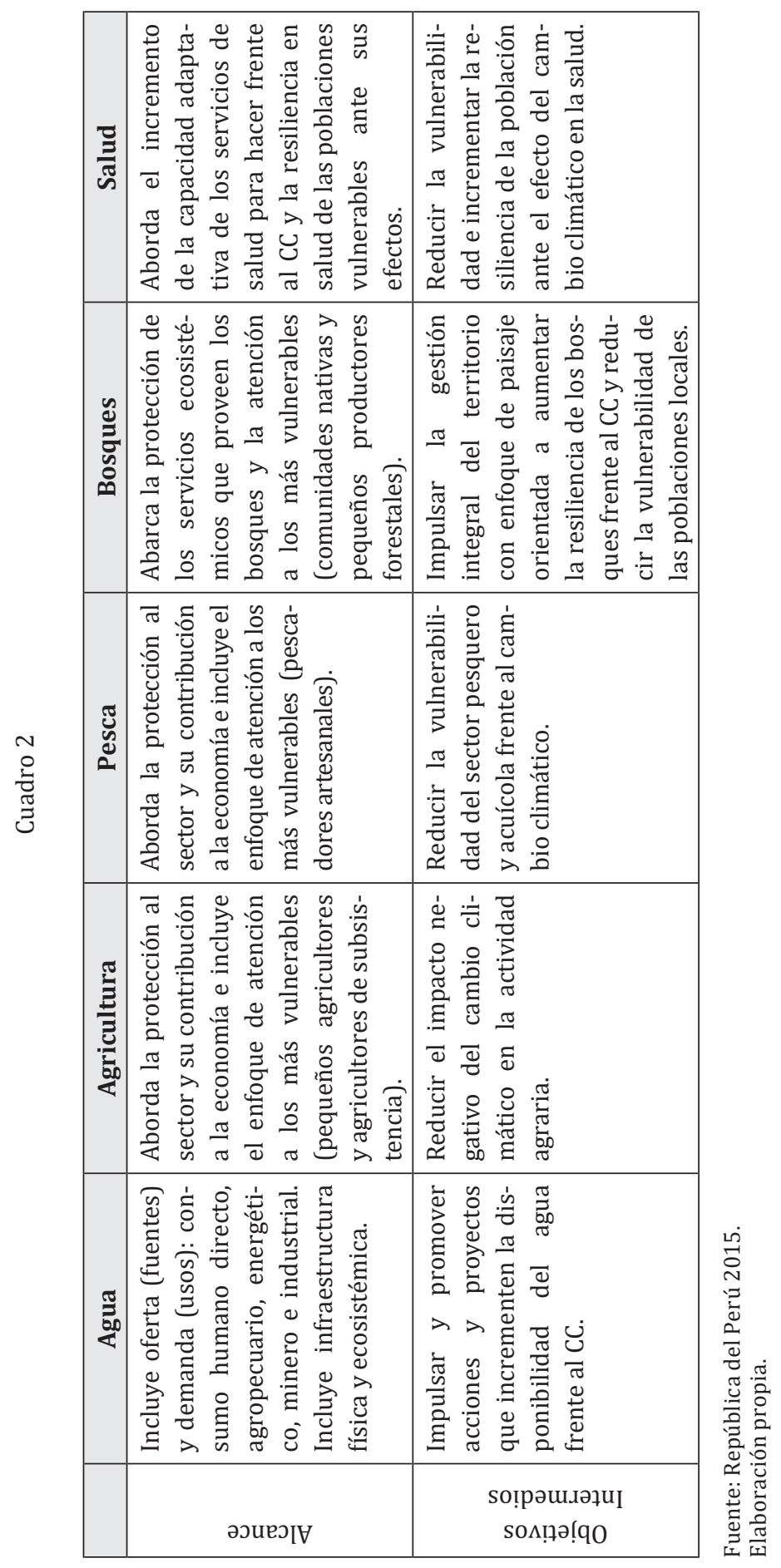


Las autoridades peruanas también han identificado cinco áreas transversales en las que se debe actuar para abordar la adaptación de manera eficaz: gestión del riesgo de desastres, infraestructura resiliente, enfoque de pobreza y poblaciones vulnerables, enfoque de género y promoción de la inversión privada en adaptación al cambio climático. Las NDC, en lo que corresponde a la mitigación y a la adaptación, no cierran los esfuerzos nacionales y constituyen pisos mínimos sobre los cuales deben impulsarse otras medidas a escala nacional (y subnacional). Cabe indicar que al momento de escribir este artículo, el Congreso de la República del Perú estaba evaluando la dación de una ley marco para la gestión del cambio climático, lo cual podría llevar a cierta reestructuración de la arquitectura de toma de decisiones gubernamentales.

\section{Las NDC y los desafíos de la política climática}

Las NDC están ligadas a los cuatros lineamientos para la construcción de una buena política climática planteados por Anthony Giddens. En primer lugar, las NDC se entienden como una política pública, lo cual implica tanto el uso de recursos públicos como la intervención articulada de los distintos niveles de gobierno para alcanzar los objetivos de la política climática; pero también implican una continuidad de dichas políticas, más allá de los gobiernos de turno. En segundo lugar, demanda un esfuerzo de convergencia de distintas políticas públicas, las cuales deben coordinarse e integrarse mutuamente, generando apoyos mutuos y sinergias mutuamente beneficiosas (Giddens 2010). En dicho contexto, las NDC no deben ser vistas solo como parte de la política ambiental; son en esencia un componente de las políticas de desarrollo sostenible. Reducir las emisiones del transporte público, por ejemplo, puede significar un sistema de transporte más eficiente, que reduce las horas que la gente pasa en el tráfico de las ciudades. «Construir una represa para almacenar agua, es también una oportunidad para mejorar la infraestructura de irrigación y mejorar la productividad de los campos. Detener la deforestación de los bosques amazónicos puede implicar a la vez generar nuevas oportunidades de aprovechamiento sostenible de los recursos forestales por parte de los pueblos indígenas que los habitan» (Lanegra 2017: 73). Es por ello que uno de los desafíos en ciernes es encaminar el proceso de implementación de las NDC y articularlo con los esfuerzos generales de promoción de desarrollo, como los Objetivos de Desarrollo Sostenible o las reformas que deberán llevarse a cabo en el marco del proceso de incorporación del Perú a la Organización para la Cooperación y el Desarrollo Económicos (OCDE), y ligar el tema climático dentro de la planificación nacional conducida por el Centro Nacional de Planeamiento Estratégico (CEPLAN). 
El tercer concepto al que están ligadas las NDC es el de la convergencia económica. De acuerdo con Giddens (2010), las innovaciones económicas y tecnológicas necesarias para combatir el calentamiento global generan a su vez ventajas competitivas para quienes las emplean; por lo tanto, no transitar hacia una economía baja en carbono - en emisiones de gases de efecto invernadero - terminaría restando competitividad a las empresas y no disminuyéndola. La OCDE, por ejemplo, «considera que en general la rigurosidad de la política ambiental — su nivel de exigencia - tiene efectos positivos sobre en la actividad económica, en particular sobre la competitividad y la innovación. Esta es una idea vinculada con la denominada hipótesis Porter-Van der Linde: una regulación estricta en sus objetivos y flexible en los caminos para alcanzarlos promueve la innovación tecnológica, lo que no solo tiene efectos positivos sobre en el ambiente, sino también sobre la competitividad de las empresas» (Lanegra 2017: 73-74). Algunos sectores productivos pueden convertirse en obsoletos como consecuencia de las ganancias de competitividad de otros. Mientras que en el corto plazo, las exigencias ambientales pueden implicar la reducción de los márgenes de ganancia de las empresas, al menos hasta que las mejoras tecnológicas compensen la pérdida o parte de ella.

Por último, las NDC están ligadas a lo que Giddens (2010) denomina el «imperativo del desarrollo». No es posible, desde su punto de vista, ignorar las aspiraciones de los países menos desarrollados como el Perú de alcanzar mejores niveles de bienestar. Si se ignora este factor, es probable que las tensiones originadas por las actuales desigualdades -entre países y al interior de ellos--, aumenten. Desde luego, la idea no es repetir el modelo insostenible en las economías emergentes. Pero esto implica un esfuerzo para desarrollar la tecnología y acceder a las fuentes de financiamiento que nos permitan alcanzar dicho objetivo. Tarea nada simple para una economía pequeña como la peruana.

\section{Los desafíos de gobernanza en la implementación de las NDC}

La implementación de las NDC requiere del actuar conjunto del sector público, del sector privado, de la sociedad civil, del sector académico y de la cooperación internacional. Sin embargo, no todos tienen los mismos incentivos para actuar. De un lado algunos actores caen en la llamada Paradoja de Giddens: «Como los peligros que representa el calentamiento global no son tangibles, inmediatos ni visibles en el curso de la vida cotidiana [...], muchos brazos se cruzarán y no harán nada concreto al respecto» (Giddens 2010: 12). Esto ocurre, según 
Giddens (2010), porque dichos actores le hacen «rebajas de futuro» a los escenarios climáticos que haga subestimar sus efectos en el largo plazo y sobrevalorar los beneficios de corto plazo. En un sentido similar, Naomi Klein (2015) piensa que todos practicamos una suerte de negacionismo climático: nos fijamos en el problema del calentamiento global un instante para luego posar la mirada en otra cuestión; o tratamos el problema con un humor evasor; o apelamos a nuestra fe en algún milagro de la tecnología; o acudimos a un impostado análisis costo beneficio que concluye que la mejor forma de adaptarnos es la acumulación de riqueza; o sentimos el problema como algo lejano y ajeno; o nos aislamos en un compromiso individual que no toca nada en las instituciones que lo rodean. Como resultado, la agenda climática se vuelve una cuestión secundaria y no prioritaria; algo que el Fenómeno de El Niño Costero de 2017 puso a la vista. Por otra parte, los políticos y los principales funcionarios de los gobiernos emiten declaraciones, directrices o definen planes o políticas, no obstante, estas no llegan a concretarse en políticas públicas eficaces. Entre tanto, las grandes empresas ignoran -y en el peor de los casos, ocultan- la evidencia y promueven sus intereses de corto plazo, a lo que debe sumarse la inevitable incertidumbre que se encuentra detrás de muchas decisiones que dependen de la construcción de escenarios siempre hipotéticos (Lanegra 2017).

Por otro lado, pareciera que muchas veces los políticos y otros intereses de corto plazo bloquean los acuerdos, en tanto ambos no ven beneficios inmediatos en las acciones que emprenden en materia climática; menos aún cuando su futuro depende de un próximo proceso electoral. Saben que los resultados de la política climática tardarán en llegar, por lo que sus éxitos serán atribuidos a otros. En cambio, si eventualmente llegara un desastre y no hubieran tomado las acciones debidas, los políticos pueden echarle la culpa a la naturaleza — la que nos castiga sin misericordia - o al destino —es una obra de Dios-; por eso, incluso con la debida información y conciencia del problema, priorizan en muchos casos la agenda de corto plazo (Lanegra 2017).

Por este motivo es indispensable que los políticos sean empujados por otros actores, cuyos intereses se mueven en un horizonte temporal más extenso, a fin de que puedan incorporar los objetivos de largo plazo. Es evidente — como destaca Naomi Klein (2015) — que la calificación y atención oficial de algo como un problema crítico, depende del poder y de las prioridades de los poderosos, y no solo de los hechos y datos empíricos; pero también puede derivar de los movimientos y la acción colectiva de la ciudadanía.

Una manera distinta es construir una capacidad de planificación sólida, apoyada por instrumentos eficaces que conlleven la necesaria alineación entre los objetivos climáticos y la planificación e inversión estatal, en todos 
los niveles. Solo un marco de planificación sólido puede reducir los incentivos de la política democrática de priorizar los objetivos electorales de corto plazo en desmedro de la política ambiental. Finalmente, como recomienda Giddens (2010), la única forma de lograr que la política climática no esté sometida al vaivén de los intereses de corto plazo es generando pactos políticos de gran amplitud que constituyan la base de una política de Estado que trascienda a los gobiernos de turno; en particular si hablamos de una democracia.

Otras dos cuestiones plantean retos enormes a los países menos desarrollados. De un lado, la gobernanza debe actuar en un contexto de debilidad estatal, entendida esta como la poca capacidad del gobierno para formular políticas y llevarlas a cabo. El Estado, indica Joel Migdal, es la capacidad de penetrar eficazmente en la sociedad que gobierna (Fukuyama 2016). Esta capacidad está definida por el tamaño de la administración pública, los recursos de que dispone - que derivan de la recaudación-, y los niveles de formación y conocimiento de los funcionarios. No obstante, también debemos considerar lo que efectivamente hacen los gobiernos (Fukuyama 2016).

Lant Pritchett, Michael Woolcock y Matt Andrews (2012) resaltan cómo los países menos desarrollados intentan copiar la organización formal de los países más desarrollados pero se muestran incapaces de generar resultados efectivos; tienen una organización pública ambiental, y políticas climáticas, pero muestran grandes problemas para convertirlas en acción. Así, el Perú puede contar con una organización ambiental y una política climática formal, pero no acompañarlas de una capacidad estatal suficiente para implementar las decisiones que se adopten. No debemos olvidar, además, que al interior de los países las diferencias de capacidad pueden ser significativas. Si es difícil impulsar una decisión en las áreas donde el Estado tiene cierta capacidad - como en las áreas urbanas-, puede convertirse en una experiencia infructuosa en las zonas rurales, en particular en las regiones altoandinas y amazónicas (Lanegra 2017).

La identificación de los problemas de capacidad exige asumir tres tareas de manera simultánea. En primer lugar, identificar el déficit de capacidades al interior de las entidades estatales, pero también con respecto al resto de actores involucrados en los procesos de gobernanza; este déficit debe ser objeto de un plan de atención de corto, mediano y largo plazo. En segundo lugar, las medidas que conforman las NDC deben considerar las capacidades actuales y el proceso de fortalecimiento señalado; este análisis debe realizarse medida por medida, en vista de la diversidad de sectores involucrados. Es necesario adecuar las políticas climáticas a dichas capacidades, a veces sincerando las metas y en otros casos estableciendo plazos de implementación más extensos, haciendo una diferenciación territorial en la ambición de las políticas o estableciendo alianzas con las comunidades y las empresas para la 
ejecución de estas políticas. En tercer lugar, las medidas deben ser ubicadas en el territorio, analizando su interacción con otros ámbitos de la acción estatal así como la de otros actores no estatales. Aquí no solo debe considerarse el campo de la legalidad, sino tomar en cuenta las complejas relaciones que en el territorio ocurren entre lo legal, lo informal o lo ilegal, que más que constituir compartimentos estancos, conforman una unidad intrincada y compleja en el Perú (CEPLAN 2016). Las autoridades deben enfrentar problemas serios de ilegalidad en sectores críticos vinculados con el calentamiento global, como la gestión de los bosques amazónicos. Tanto los informales —abiertamentecomo los ilegales — de forma subrepticia-, pueden oponerse a reformas que consideran pueden poner su actividad ilegal en peligro, con el apoyo incluso de las propias autoridades, lo cual debe entenderse dentro del contexto de un país con alto grado de corrupción. Sin embargo, tanto la ausencia de una política territorial fuerte, como los problemas enfrentados en la lucha contra la corrupción y las limitaciones de las políticas de formalización (en minería y en el área forestal) plantean un escenario cuesta arriba para la implementación de las NDC.

\section{A modo de conclusión: algunas líneas de acción}

Más allá de las decisiones ya adoptadas para el proceso de definición de las medidas de mitigación y adaptación para el cumplimiento de las NDC, lo arriba descrito muestra que el principal desafío lo representan los obstáculos que pueden presentarse al momento de la implementación; en particular, por las debilidades en las capacidades de los actores involucrados y por la ausencia de una política territorial efectiva. Esto, desde luego, no olvida lo fundamental que es atender las cuestiones del financiamiento y la transferencia tecnológica, pero advierte que incluso en el caso de que ambas esferas obtengan respuestas satisfactorias, la implementación puede verse limitada por los problemas de gobernanza. Desde luego, será necesario estudiar el proceso de implementación para llegar a conclusiones más sólidas al respecto, pero sí es importante contar con un marco de análisis que les permita a las autoridades optar por las vías de acción más adecuadas considerando la información existente.

El Proyecto de Apoyo a la Gestión del Cambio Climático — una iniciativa apoyada por la Cooperación Suiza para el Desarrollo y ejecutada por Libélula-, por ejemplo, viene planteando la necesidad de considerar en dicho análisis no solo la definición precisa de las medidas a adoptar, sino también una estructura de coordinación y liderazgo que conduzca un proceso de implementación que no solo incorpore al sector público, sino también al sector privado, a la sociedad 
civil, a la academia y a la cooperación internacional. Es una propuesta que entiende que muchas medidas requerirán de un activo compromiso político que impulse su desarrollo, así como de una red de soporte que evite desviaciones inconvenientes. Del mismo modo, el análisis no se limita a la implementación de la medida misma, también considera las acciones o proyectos que permitan atender otras dimensiones como las capacidades institucionales, el marco normativo, la identificación de los actores, alianzas y coaliciones a favor - 0 en contra- de las medidas, así como las necesidades de financiamiento. Todo ello debe conducir a una estructura de implementación que permita entender y conducir el proceso, lo cual puede llevar a la identificación de las prioridades así como al aprovechamiento de las sinergias entre las medidas; asimismo, permitiría poner en relieve las eventuales incompatibilidades entre las mismas y otras políticas estatales. Desde luego, esta propuesta - y otras que surjan - deberán ser objeto de mejora y corrección conforme avance el proceso de implementación de las medidas que conduzcan a alcanzar las metas de las NDC. Esto dependerá también de los avances en la atención de los problemas generales de gobernanza del país que hemos también descrito. 


\section{Referencias}

Andrews, Matt, Lant Pritchett, y Michael Woolcock (2012) Escaping Capability Traps through Problem-Driven Iterative Adaptation (PDIA). CGD Working Paper 299. Washington, D.C.: Center for Global Development. http://www.cgdev.org/ content/publications/detail/1426292.

Centro Nacional de Planeamiento Estratégico (2016). Economía informal en Perú: Situación actual y perspectivas. Lima: CEPLAN.

Funuyama, Francis (2016). Orden y decadencia de la política. Desde la Revolución Industrial hasta la globalización de la democracia. Barcelona: Deusto.

GEARY, Mirta. (2016) «Debatiendo el concepto de valor público en el marco de las políticas ambientales». En: Andrea Lampis (editor). Cambio ambiental global, Estado y valor público: La cuestión socio-ecológica en América Latina, entre Justicia Ambiental y «Legítima depredación». Bogotá: Universidad Nacional de Colombia, INTE-PUCP, CLACSO, pp. 27-43.

GIDdens, Anthony (2010). La política del cambio climático. Madrid: Alianza Editorial.

KLEIN, Naomi (2015). Esto lo cambia todo. El capitalismo contra el clima. Barcelona: Paidós.

LANEGRA, Ivan (2017). ¿Qué es el cambio climático? Calentamiento global y sociedad. Lima: Planeta.

Organización de Naciones Unidas (ONU) (2015). Acuerdo de París. Disponible en: https://unfccc.int/sites/default/files/spanish_paris_agreement.pdf

Porras, Francisco. Gobernanza. Propuestas, límites y perspectivas. Ciudad de México, Instituto Mora, 2016.

Postigo, Julio (editor) (2013). Cambio climático, movimientos sociales y políticas públicas. Una vinculación necesaria. Lima: CLACSO, INTE-PUCP, ICAL.

REPÚBLICA DEL PERÚ (2015). Contribución prevista y determinada a nivel nacional (INDC) de la República del Perú. Septiembre 2015. Disponible en: http:// www4.unfccc.int/ndcregistry/PublishedDocuments/Peru\%20First/ iNDC\%20Per\%C3\%BA\%20castellano.pdf

SACHS, Jeffrey D. (2015). La era del desarrollo sostenible. Nuestro futuro está en juego: incorporemos el desarrollo sostenible a la agenda política mundial. Barcelona: Ediciones Deusto.

Proyecto de Apoyo a la Gestión del Cambio Climático (2016) Preparando el terreno para la Contribución Nacional. Presentación preliminar de Resultados Informe de Taller de Trabajo 7 de diciembre del 2016. Lima: Libélula Instituto para el Cambio Global. Disponible en: http://proyectoapoyocambioclimatico.pe/wpcontent/uploads/2017/03/Reporte-Final-PDF.pdf 
